\title{
Detection of false command and response injection attacks for cyber physical systems security and resilience.
}

\author{
EKE, H., PETROVSKI, A. and AHRIZ, H.
}




\section{Detection of False Command and Response Injection Attacks for Cyber Physical Systems Security and Resilience}

\author{
Hope Eke E $^{*}$ \\ h.eke@rgu.ac.uk \\ School of Computing, \\ Robert Gordon University \\ Aberdeen, Scotland, UK
}

\author{
Andrei Petrovski \\ a.petrovski@rgu.ac.uk \\ School of Computing, \\ Robert Gordon University \\ Aberdeen, Scotland, UK
}

\author{
Hatem Ahriz \\ h.ahriz@rgu.ac.uk \\ School of Computing, \\ Robert Gordon University \\ Aberdeen, Scotland, UK
}

\begin{abstract}
The operational cyber-physical system (CPS) state, safety and resource availability is impacted by the safety and security measures in place. This paper focused on i) command injection (CI) attack that alters the system behaviour through injection of false control and configuration commands into a control system and ii) response injection (RI) attacks that modifies the response from server to client, thereby providing false information about system state. In this project, we implemented deep learning (DL) multi-layered security model approach for securing industrial control system (ICS) against malicious $\mathrm{CI}$ and RI attacks. We validated this approach with two case studies: i) network transactions between a Remote Terminal Unit (RTU) and a Master Control Unit (MTU) in-house SCADA gas pipeline control system and ii) a case study of command and response injection attacks. Based on this project result, we show that the proposed approach achieved a significant attacks detection capability of $96.50 \%$. Also, demonstrated that performance of attack detection techniques applied can be influences by the nature of network transactions with respect to the domain of application. Hence, robustness and resilience of operational CPS state and performance are influenced by the safety and security measures in place which is specific to the CPS device in question.
\end{abstract}

\section{CCS CONCEPTS}

- Security and privacy $\rightarrow$ Distributed systems security.

\section{KEYWORDS}

Advanced Persistent Threats(APTs), Command and Response Injection, Cyber-Physical Systems (CPSs), Industrial Control Systems (ICS), Distributed Control System (DCS), Supervisory Control and Data Acquisition (SCADA)

\section{INTRODUCTION}

Critical infrastructure are CPS that are responsible for maintaining normal industrial plants operation such as gas pipelines, water treatment and power plants. The CPS are control systems that composed of interconnected computer-based networked and integrated cyber physical components that monitor, control and exchange vital information through physical components [16], [17]. These physical components consist of sensors, controller and actuators that aids communicate [17]. Examples of such systems are Distributed Control System (DCS) and Supervisory Control and Data Acquisition (SCADA) that contain control systems which are used in nuclear power plants [13], water and sewage systems, and irrigation systems [11]. Figure 1 is a representation of simple SCADA system. The computer systems used to control physical functions of the operating systems are not immune to the threat of today's sophisticated cyber attacks and can be potentially vulnerable [16]. Potential



Figure 1: A Simple Scada System

threats can affect ICS device at different level; hence security of each component is extremely important to avoid compromised to any component [9]. ICS also utilizes diverse communication platforms and protocols to increase efficiency, productivity reduce operational costs and further improve organization's support model [21].

The complexity of the ICS architecture, and the increased efforts of control physical functions in processing and analysing data, has lead to increased interactions between control network and business network [21] - [19]. The possibility of attacks on control systems in order to gain access to the physical part of ICS and daily operational challenges due to these increased cyber-physical interaction are on the high side [11] and [19].

Hence, ensuring the security of these systems are of very important to avoid any operational disruption. However, this requires a complex approach to identify and mitigate security vulnerabilities or compromise at all levels within the ICS to maintain resource 
availability, system safety, integrity, confidentiality and authentication against the most dangerous type of attack known as APTs [2] and malicious injection attacks.

Due to increased efforts in ICS's data analytics, interaction between control network and business network, software usage has also grown and adapted to new environments at a significant pace. This technology advancements has greatly changed how we interact with the connected world, as a result of these new technological advancements, threat actors have also embraced and adapted to this development to improve their own strategy in creating new sophisticated malicious applications as highlighted in [21] and [12].

There is a clear need for intrusion detection / protection system specifically for critical infrastructures (CIs) that can significantly improve the security of such systems. Any compromise to these systems caused by intrusion attacks can lead not only to disruption of operation, huge financial loses but, more importantly, the risk to the public safety.

The contribution of this paper can be summarised as follows:

- We propose an ensemble deep learning multi-layered security detection approach for ICS devices.

- We implemented a multi-layered security detection approach that takes into consideration the distributed and multi-level nature of ICS architecture and reflect on the four main SCADA cyber attacks.

- The achieved results suggest that the proposed approach possess attack detection capability and demonstrated that performance of attack detection techniques applied can be influences by the nature of network transactions with respect to the domain of application.

\subsection{Paper Organisation}

The remainder of the paper is organised as follows. Section 2 contains a brief discussion of related work geared toward the security of CPS. In section 3 detailed description of our approach is discussed. Experiments, evaluation metrics and analysis results are discussed in Section 4. Section 5 and 6 presents the conclusion of this paper and our future work respectively.

\section{RELATED WORK}

This section contains a brief discussion of related work to support the maintaining security and stability of CPS. The ability to detect every possibilities of an active attack on an active CPS is a global security challenge. There have been a number of successful breaches of critical infrastructure. Stuxnet is one example of a sophisticated targeted cyber attack purposefully launched to target critical nuclear infrastructure in Iran as highlighted in [4] and [3].

However, the four main recorded targeted attacks malware tailored against ICS are STUXNET, BLACKENERGY 2, HAVEX and CRASHOVERRIDE, where STUXNET is the first ever recorded for disrupting physical industrial processes while CRASHOVERRIDE is the second and also the first known to specifically target the electric grid [20] and [22]. CRASHOVERRIDE is not unique to any vendor or configuration but utilises the knowledge of grid operations and network communications to cause disruption resulting to electric outages and is not necessarily malware for espionage [15] and [10].
Numerous techniques have been proposed and successfully implemented to detect these type of attacks. However, most of these proposed works has led to a significant pool of solutions geared towards addressing securing the CPS [5]. One of this threat detection model in a specific critical infrastructures was carried out by Linda et al. in [16] using a hybrid of two neural network learning algorithms - the Error-Back Propagation and Levenberg-Marquardt, for normal behavior modeling to develop an IDS using Neural Network based Modeling (IDS-NNM). This IDS-NNM was achieved by developing window based feature extraction technique; construction of training dataset using randomly generated intrusion vectors from real network data.Their result shows the ability of IDS-NNM to detect long and short intrusion attempts consisting of several packets and achieved good detection rate while generating no false positives when evaluated with previously unseen testing data.

Work in [17] which focused on generating a cyber defense system that will maximizes the likelihood that a cyber-physical system under sensor attack can still recover and reach the desired state without a noticeable performance degradation using combination of watermarking and resilient kalman filtering (RKF) technique accompanied with a watermarking approach.

Also, in [1], the author considered a case of nonlinearities in communication data flow in Automatic Generation Control (AGC) system, applied stacked RNN-LSTM model as a detector and classifier in order to detect False Data Injection (FDI) attacks in AGC systems and achieved accuracy of $94 \%$. The authors focused on three types of attack; the ramp, step and pulse attacks since attacks in AGC target frequency deviation signals and tie-line power signals. Accuracy, sensitivity, specificity and precision were calculated as metrics measure in order to validate their proposed model detection classification capability. Two case scenarios were implemented using RMSprop optimizer to enhance the model performance and archived a precision of $99.01 \%$ and $99.22 \%$.

We implemented a multi-layered security detection approach that takes into consideration the distributed and multi-level nature of ICS architecture and reflect on the four main SCADA cyber attacks using ensemble DL model on network transactions between RTU and MTU. We expect that the ideas behind this analysis approach as presented in this paper will aid in the efforts to improve and maintain the security of cyber-physical systems stability.

\section{APPROACH}

The ability to maintain the authentic communication settings such as pressure set point, proportional integral derivative (PID) parameters, or relief valve control state within a SCADA system is a very critical issue.

SCADA systems has a regular set of read and write commands communication patterns that are repeated in a loop. For instance, the system command writes the contents of all registers and coils used, then the measured state of the system is read by the Modbus read holding register command. Each of these commands are followed by response. These operations are performed by programmable logic controller (PLC) and human machine interface (HMI) using a standard SCADA communication protocol. The PLC controller reads inputs, analyse current state, calculate responses, 
and write outputs while the HMI monitor and control the physical process remotely.

To execute CI attacks, the attackers maliciously inject a command into the system server that modifies settings such as pressure set point, PID within a SCADA system. On the other hand, the RI attacks then alters the response coming from the server to client thereby provide false system state information [18]. We have exploited the regular communication patterns in SCADA system using deep leaning algorithms that has a nonlinearity feature to build a model of normal behaviour and detect abnormal deviations

\subsection{Our proposed model operates in three layers}

(1) Data input and probing layer

(2) Data analysis Layer

(3) Decision Layer (uses voting and probability confidence)

3.1.1 Data input and probing layer: consists of two modules; (i) Data input which involves data gathering and raw sample / simulated synthetic data been introduce into the system and (ii) Probing Module - this involves data preprocessing the collected data.

- Normalisation - ZScore method of standardisation is used to normalise all numerical features to preserve the data range and introduce the standard deviation and variance to improves model convergence speed during training.

$$
Z=\frac{x-\mu}{\sigma}
$$

Where $Z$ is ZScore, $x$ is the individual data point, $\mu$ is the mean of $x$ for a given attribute; that is uniform real number between 0 and 1, represented as Equation 2

$$
\mu=\frac{1}{n} \sum_{i=1}^{n} x_{i}
$$

and Rho $(\sigma)$ is the standard deviation as represented in Equation 3

$$
\sigma=\sqrt{\frac{\sum(x-\mu)^{2}}{n}}
$$

3.1.2 Data analysis Layer: The rate of attack detection is affected by the parameters used as this parameters have direct impact on attack detection, based on this, several experiments with different network configuration were implemented to find the best optimal values for parameters such as learning rate, network structure such as number of hidden recurrent layer

The processed data are passed to this layer, data is analysed, attack(s) are distinguished from normal activities, taken note of the identified issued with class distribution and classifying rare attacks. Result from this layer is passed to decision engine layer.

3.1.3 Decision Layer: This Layer operates using three approaches; first, it receives information from analysis layer, extract the attack step present. Secondly, it processes this information and links attacks steps that are related. Lastly, it then uses voting and probability confidence to check if the attack is a potential chain of attack campaign is found and if is consistent with other attack campaigns.

\subsection{Recurrent Neural Network}

The Decision Layer is implemented with ensemble RNNs variants for data analysis. RNN is an effective class of artificial neural network (ANN) used when dealing with sequence data. The RNN uses previous inputs and outputs to adjust the weights of the networks, thus creating a memory to improve its performance [14] It maps a sequence of inputs to the outputs thereby controlling the information flow signal with respect to time. Hence, RNNs is suitable for dynamic real time network pattern analysis with time.

The formulated mathematical representations of the RNN computational flow are represented in Equation 4

$$
s_{t}=f_{w}\left(s_{t-1}, x_{t}\right)
$$

where $f w$ is the recursive function. Given that a network has an input layer $x$, hidden layer $s$ and output layer $o$. The current observed input to the network at time step $t$ is denoted as $x_{t}$, the hidden state at time step $t$ is denoted as $s_{t}$ and $s_{t-1}$ represent the previous hidden state.

Each hidden layers has a sigmoid activation function which can be calculated with Equation 5, this function is applied to produce non-linearity value by transforming the input into values usable by the output layer.

$$
\operatorname{sigmoid}(x)=\frac{1}{1+e^{-x}}
$$

Based on the pre-configured number of time steps during training, the network screens the sequence data by creating a time window to identify a temporal pattern across the features and labels within this window. The same process is repeated within the network by sliding over the entire data sequence while updates the weights accordingly. With the assumption that parameters share the same weight $W$ across the whole sequence in each time step t reducing the amount of parameter to be trained, this assumption is utilised to compute the gradient for weight parameter across time step $t$. Hence at a given time $t$, we have;

$$
\begin{gathered}
s_{t}=\tanh \left(W_{x s} x_{t}+W_{s s} s_{t-1}+b_{s}\right) \\
s f=\frac{e^{x_{t}}}{\sum_{j=1}^{n} e^{x_{J}}}
\end{gathered}
$$

where $s_{t}$ is the calculated hidden layer, $\tanh$ is the hidden layer nonlinear activation function, softmax function $s f$ represented in Equation 7 is used at the last layer in Equation 8. the weight shared among the previous hidden state $s_{t-1}$, input state $x_{t}$ and output $o_{t}$ across all the time sequence $t$ are the weight shared between the hidden $s$ and output o across all the time sequence are $W_{s s}, W_{x s}$ and $W_{o s}$ respectively, while $b_{s}$ and $b_{o}$ are the bias terms for the hidden state and output $o$ at time step $t$.

$$
o_{t}=\operatorname{softmax}\left(w_{s o} s_{t}+b_{0}\right)
$$

In order to minimise the cumulative network error, the loss, or error as applied on this study is the cross-entropy loss which is the sum of all input-output pairs errors in a sequence over all the time steps $t$, is referred to as the loss $\mathrm{L}$ function, given as Equation 9,

$$
L(o, y)=-\sum_{t} y_{t}(x) \log _{t}(x)
$$

where $y$ is the true probability distribution, $o$ is the predicted probability distribution, $y_{t}$ is the actual true probability distribution 
class value at time step $t$ and $o_{t}$ is the actual true predicted class distribution value at time step $t, x$ is an input and $\log$ is natural $\log$.

To derive the derivative of Equation9 with respect to the actual true predicted class value $o_{t}$ as calculated with Equation 8, assume $L$ as the given objective function, $L(t)$ as output at current hidden time $t$ and $L(t+1)$ as the output at the previous hidden state time $t+1$. Then, we have;

$$
\frac{\partial L}{\partial o_{t}}=-\sum_{t} y_{t} \frac{\partial \log o_{t}}{\partial s_{t}}=-\sum_{t} y_{t} \frac{1}{o_{t}} \frac{\partial o_{t}}{\partial s_{t}}
$$

Then applying the chain rule to derive the gradient of the $s f$ from Equation 8, we arrive at Equation 11.

$$
\frac{\partial L}{\partial o_{t}}=-\left(y_{t}-o_{t}\right)
$$

Since the hidden state and output share the same weight $W_{\text {so }}$ across the whole sequence in each time step $t$ reducing the amount of parameter to be trained, weight can be differentiated at each time step $\mathrm{t}$ as shown from Equation 12 to 17.

$$
\frac{\partial L}{\partial W_{s o}}=\sum_{t} \frac{\partial L}{\partial o_{t}} \frac{\partial o_{t}}{\partial W_{s o}}
$$

Then, we derive the gradient with respect to each bias $b_{o}$ unit to achieve Equation 13,

$$
\frac{\partial L}{\partial b_{o}}=\sum_{t} \frac{\partial L}{\partial o_{t}} \frac{\partial o_{t}}{\partial b_{o}}
$$

Also, to derive the gradient with respect to weight $W_{s s}$, we consider the previous time step

$$
t \rightarrow \mathrm{t}+1
$$

and arrived at Equation 14

$$
\frac{\partial L(t+1)}{\partial W_{s s}}=\frac{\partial L(t+1)}{\partial o_{t+1}} \frac{\partial o_{t+1}}{\partial s_{t+1}} \frac{\partial s_{t+1}}{\partial W_{s s}}
$$

Considering that weight $W_{s s}$, and $W_{o s}$ shared across all the time $t$ sequence are similar. Likewise, in RNN model, the calculation of the subsequent state hidden state $s_{t}$ depends partially on the previous hidden state $s_{t-1}$. Hence, we arrived at Equation 15

$$
\frac{\partial L(t+1)}{\partial W_{s s}}=\frac{\partial L(t+1)}{\partial o_{t+1}} \frac{\partial o_{t+1}}{\partial s_{t+1}} \frac{\partial s_{t+1}}{\partial s_{t}} \frac{\partial s_{t}}{\partial W_{s s}}
$$

We arrived at Equation 13 (15) by clustering the weight over the whole sequence using the Backpropagation Through Time (BPTT) from time step $t$ to 0 with respect to $W_{s s}$, we get Equation 16 ,

$$
\frac{\partial L(t+1)}{\partial W_{s s}}=\sum_{t} \sum_{k=1}^{t+1} \frac{\partial L(t+1)}{\partial o_{t+1}} \frac{\partial o_{t+1}}{\partial s_{t+1}} \frac{\partial s_{t+1}}{\partial s_{k}} \frac{\partial h_{k}}{\partial W_{s s}}
$$

Taking the gradient with respect to $W_{x s}$ over the whole sequence, we applied the same process as from Equation 12 to 17.

$$
\frac{\partial L(t+1)}{\partial W_{x s}}=\sum_{t} \sum_{k=1}^{t+1} \frac{\partial L(t+1)}{\partial o_{t+1}} \frac{\partial o_{t+1}}{\partial s_{t+1}} \frac{\partial s_{t+1}}{\partial s_{k}} \frac{\partial h_{k}}{\partial W_{x s}}
$$

For more understanding of how these equations are derived, please check Hagan et al, in [7]

\section{EXPERIMENT}

The purpose of this study is to examine the performance of implementing deep DL ensemble stacked RNN variants approaches to detection false command and response injection attacks. We have used network typologies and payload information values of The New Gas Pipeline Dataset containing 214,580 Modbus network packets with 60,048 packets that are associated with a cyber attack. These attacks are categorised into 7 different attack categories with 35 different specific type of attacks [8] and [9]. These attack categories are narrowed down to four overall categories as follows: Response injection (RI) attacks, Command injection (CI) attacks, Denial of Service (DoS) attacks and Reconnaissance. Figure 2 shows the number of records in each of the categories.

Two different tasks were implemented. The first task focused on deriving hyper-parameter values for best performance model. In the second task, the best hyperparameter values were implemented in measuring the model performance. All the standard data mining processes such as data cleaning and pre-processing, normalisation, visualisation and classification were implemented in Python. The batch size of 124 and epochs are run up to 500 with a learning rate set in the range of $0.01-0.5$ on a GPU-enabled TensorFlow network architecture. All the 17 features were used as input vector with $70 \%$ as training set and $30 \%$ as validation set for the multi attack classification. The training dataset were normalised from 0 to 1 . This was trained using sigmoid activation function through time with ADAM optimiser, sigmoid function was used on all the three gates and categorical cross entropy as loss function for error rate.

Also, these tasks were carried out with traditional machine learning (ML) classification algorithms - Decision Tree (DT). The ML classification result was compared to Deep ensemble RNNs-CNN result in order to further evaluate the detection capability of used approach and report the result in Table 1 and 2 .

\subsection{Experimental Data}

Figure 2 is the visualisation representation of The New Gas Pipeline data classes records.

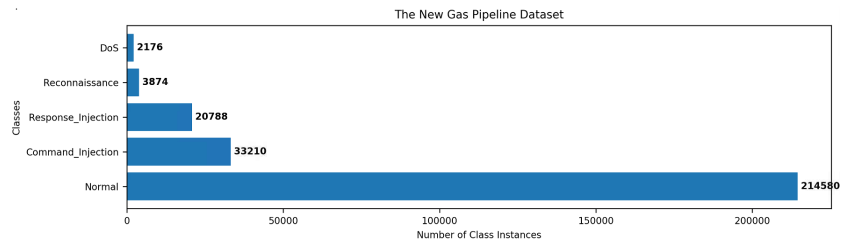

Figure 2: Four Main Attack Group and Normal Classes

\subsection{Hyperparameters settings used}

- Batch sizes: 32, 64 and 128

- Learning rate: 0.0002 to 0.00005 with polynomial decay over all the epochs.

- No of iterations: 100 - 500epochs.

- Neural network: Four layers were used

- Each of the hidden layers has a sigmoid/ReLU activation function applied to it to produce non-linearity. This transforms the input into values usable by the output layer. 
- The softmax function is applied to the output layer to get probabilities of categories. This also helps in learning with cross entropy loss function.

- Adaptive Moment Estimation (Adam) optimiser is used for the back propagation to minimise the loss of categoricalcross entropy.

- The dropout is used to alleviate the over-fitting (used as regularization technique used to prevent over-fitting in Neural Networks. This randomly removes the units along with connections.

\subsection{Evaluation Metrics}

Generally, accuracy is used as a traditional way of classification performance measure. This matric measure is no longer appropriate when dealing with multiclass imbalance data since the minority class has little or no contribution when compared to majority classes toward accuracy [23]. For this reason, this study considered precision, recall, f1-score, overall accuracy, area under the curve $(A U C)$ receiver operating characteristic $(R O C)$ and confusion matrix are used to validate the approach of using $\mathrm{RNN}$ variants for detecting false command and response injection attack and get a clearer understanding of the output. These metrics are calculated based on True Positive $(T P)$, True Negative $(T N)$, False Positive $(F P)$, and False Negative $(F N)$.

(1) $T P$ - abnormal instances correctly predicted as abnormal.

(2) $T N$ - normal instances correctly predicted as normal

(3) $F P$ - normal instances incorrectly predicted as abnormal

(4) $F N$ - abnormal instances incorrectly predicted as normal

(5) Precision $(P)$ - is the ability of a classification model to identify only the relevant data points, that is the ratio of $T P$ records over the sum of $T P$ and $F P$. Represented as Equation 18

$$
P=\frac{T P}{(T P+F P)}
$$

(6) $\operatorname{Recall}(R)$ - which is also known as probability of detection, true positive rate TPRor sensitivity $S$ is the ability of a model to find all the relevant cases within data, that is the ratio of the $T P$ records over the sum of $T P$ and $F N$. Represented as Equation 19.

$$
R=\frac{T P}{(T P+F N)}
$$

(7) $f 1$ - score $(f 1)$ - is the weighted average of precision and recall, that is the harmonic mean of precision and recall of a class in one given metric, represented as in Equation 22, where weight parameter is denoted by $\beta^{2}$ generally set to 1 by default and which measures the trade-off between recall and precision. Represented as Equation 20.

$$
f 1=\frac{\left(1+\beta^{2}\right) \cdot \text { precision.recall }}{\beta^{2} \text { precision }+ \text { recall }}
$$

(8) AUC - ROCcurve - The precision-recall curve shows the trade-off between precision and recall at different threshold settings, where AUC measures the degree of separability and $R O C$ represent the probability curve and also a useful technique for model output visualising, organising and selecting classifiers based on their performance as described in [6]. A high area under the curve represents both high recall and high precision, where high precision relates to a low false positive rate, and high recall relates to a low false negative rate. High scores for both precision and recall show that the model is returning accurate results $P$, as well as returning a majority of all positive results $R$. An ideal model with high precision and high recall will return many results, with all results labelled correctly. The AUC-ROC curve can also be used as a scaler measure rather than the higher the AUC value, the better the model [8].

$$
A U C=\frac{1+T P_{\text {rate }}-F P_{\text {rate }}}{2}
$$

(9) OverallAccuracy $(\mathrm{OaAcc})$ - Measures the rate of the correctly classified class instances of all the classes (attacks and normal). An overall classification performance is an important performance matrix require to evaluate the overall model performance rate, calculated as represented in Equation 22.

$$
O a A c c=\frac{(T P+T N)}{(T P+T N+F P+F N)}
$$

\subsection{Results and Discussions}

To validate the approach of using the RNNs-CNN model for detecting attacks statistical matrices such as true positive rate (TPR), false positive rate (FPR), $P, R$, and $f 1$, are calculated (i) to evaluate the ability of this approach to accurately detect and classify an attack as abnormal and also (ii) to check the ability of this model to detect different type of attacks accurately while focusing on CI and RI attacks.

Table 1 contains the comparative summary result of the individual algorithms score, while Table 2 shows the overall of the study approach performance score in detecting malicious $\mathrm{CI}$ and RI attack groups. In this study, RNNs-CNN model was used as classifier and detector and were able to achieve a significant result of $86.20 \%$ overall average accuracy. Although, a closer observation of the individual performance of each of the algorithm, LSTM shows a better detecting rate for the CI and RI of this study interest with $93.30 \%$ and $92.04 \%$ respectively. We went further to implement the same approach using ML algorithm "DT", this turns out to yield slightly higher score of $95.53 \%$ as in Table 2. Considering other evaluation metrics used, our approach achieved a $P$ of $96.41 \%$ and Sensitivity of 99.71 .

Furthermore, the validation accuracy, loss rate and AUC-ROC shows a clear view of the overall performance, the error rate and accuracy validation as can been seen in can be visualised in Figures 8 - 16. However, there are some spikes in the validation accuracy and loss as shown in Figures 9-16, following the individual model detection capability and loss per epoch, with micro and macro-average ROC curve of $91 \%$ and $72 \%$ respectively obtained, it indicates that the classifier performs well for each individual class.

- Confusion Matrix: Confusion Matrix: Figure 3-7 are the individually generated confusion matrix to show the predicted and the actual true detection of all the four attacks group for each of the algorithms used. Figure 2 shows the number of instances of the CI, DoS, Normal, Reconnaissance and RI, where DoS appear to have a fewer connection records while normal contains the highest connection records. The model 
Table 1: Overall Performance for All Attacks Detection

\begin{tabular}{|l|l|}
\hline Criteria & Score \\
\hline Recall & 86.1 \\
\hline Precision & 87.9 \\
\hline f1-score & 82.1 \\
\hline TPR & 96.5 \\
\hline FPR & 0 \\
\hline Validation Loss & 32.6 \\
\hline Detection rate & 86.1 \\
\hline Overall Score \\
\hline OaAcc (\%) & 86.2 \\
\hline micro f1 (\%) & 91 \\
\hline macro f1 (\%) & 72 \\
\hline \multicolumn{2}{|l}{}
\end{tabular}

Table 2: Comparative Result for CI / RI Attacks Detection

\begin{tabular}{|l|l|l|l|l|l|l|l|l|l|l|}
\hline Algorithm & \multicolumn{2}{l}{ LSTM (\%) } & \multicolumn{2}{l|}{ RNN (\%) } & \multicolumn{2}{l|}{ GRU (\%) } & \multicolumn{2}{l|}{ CNN (\%) } & \multicolumn{2}{l|}{ DT (\%) } \\
\hline Criteria & $C I$ & $R I$ & $C I$ & $R I$ & $C I$ & $R I$ & $C I$ & $R I$ & $C I$ & $R I$ \\
\hline Precision & 96.41 & 76.54 & 95.73 & 100 & 96.47 & 89.92 & 96.47 & 89.92 & 97.77 & 72.4 \\
\hline Specificity & 99.71 & 99.95 & 99.65 & 100 & 99.7 & 99.98 & 99.7 & 99.98 & 99.68 & 97.91 \\
\hline Detection rate & 93.3 & 92.04 & 93.09 & 92.03 & 93.53 & 92.07 & 93.53 & 92.08 & 99.17 & 95.53 \\
\hline
\end{tabular}

tends to learn the class with more records properly without confusing their identity unlike those with fewer connections records. This indicates data imbalance distribution problem.

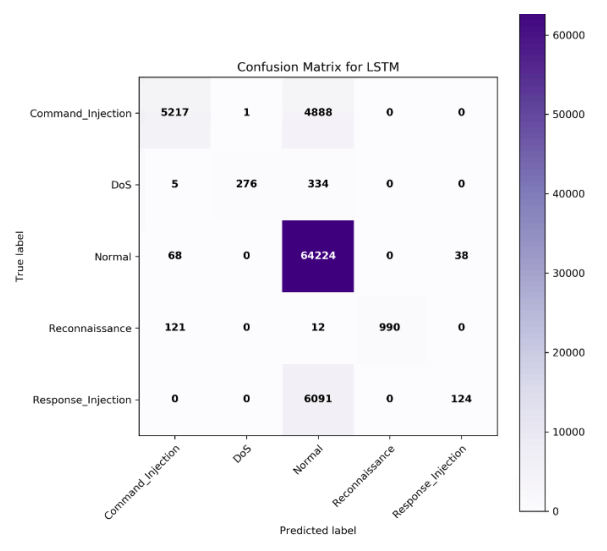

Figure 3: Multi-Classification Confusion Matrix for LSTM

\subsubsection{Visual Representations of Experimental Confusion Matrices:}

\section{CONCLUSION}

In this study, we implemented a multi-layered security detection approach based on Deep Leaning (DL), that takes into consideration the distributed and multi-level nature of ICS architecture and reflect on the four main SCADA cyber attacks. We went further to implement attacks classification with ML "DT" classifiers as contained in Table 1 and 2. The result of the implemented algorithms achieved a competitive overall accuracy and detection rate with $0 \%$ FAR and TPR of $96.50 \%$. We also noticed a higher detection rate of CI to RI from all the individual algorithms implemented, although

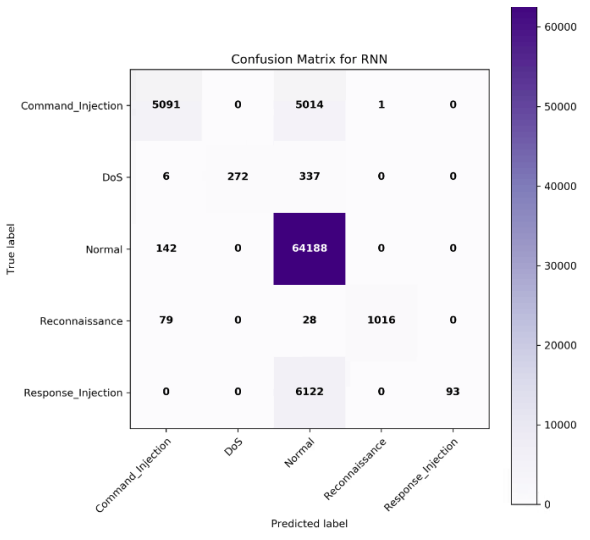

Figure 4: Multi-Classification Confusion Matrix for RNN



Figure 5: Multi-Classification Confusion Matrix for GRU

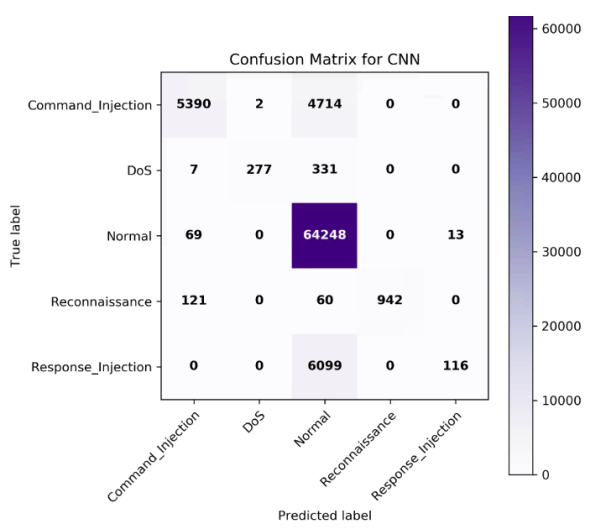

Figure 6: Multi-Class Confusion Matrix for CNN

CI has more connection records, the implemented approach appear to be more suitable for classifying high-frequency attacks. The percentage detection rate achieved as represented in Table 2 shows a slight detection capability for ML than DL for this study, however, the overall result suggests that the RNNs-CNN model is a good candidate for developing attack detection systems. 


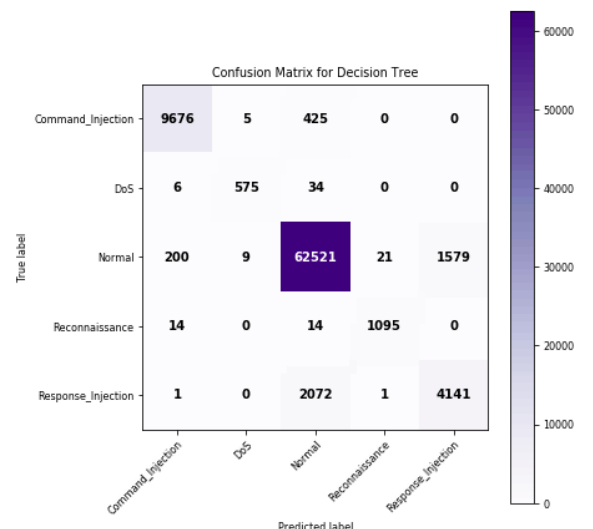

Figure 7: Multi-Class Confusion Matrix for DT

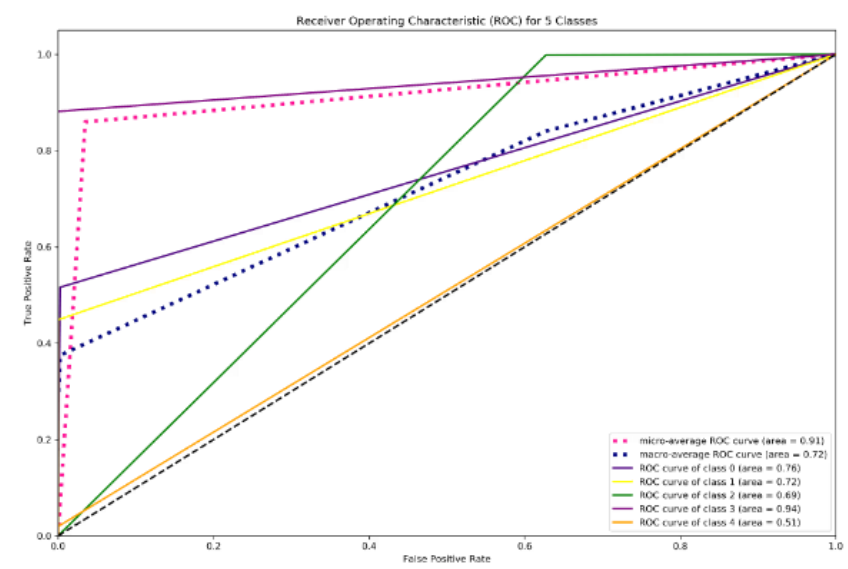

Figure 8: AUC-ROC graph for all five Classes

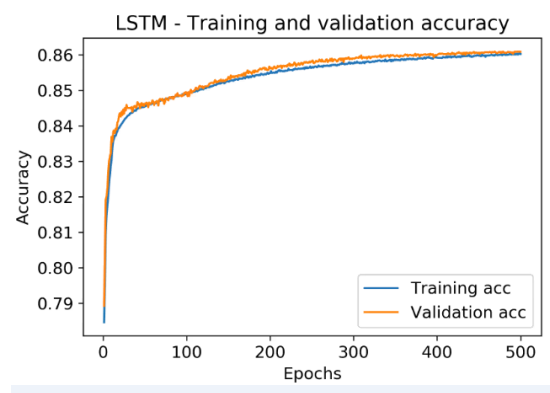

Figure 9: Validation accuracy against epochs for LSTM

\section{FUTURE WORK}

This work on the implementation of an ensemble DL multi-layered security detection approach on ICS device using The New Gas Pipeline (NGP) dataset is an ongoing study. Further work will explore full implementation of APT Detection Framework Based on Deep APT Step Analysis \& Correlation (APT-DASAC) on NGP and



Figure 10: Validation accuracy against epochs for RNN

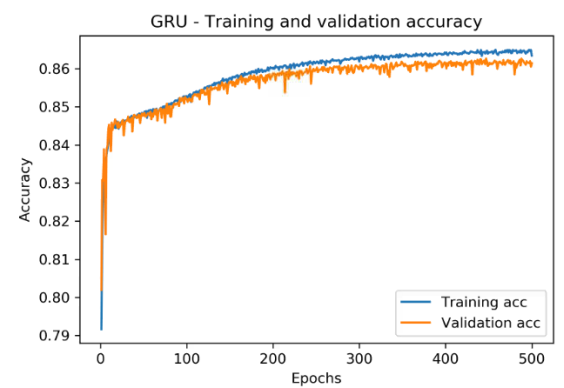

Figure 11: Validation accuracy against epochs for GRU

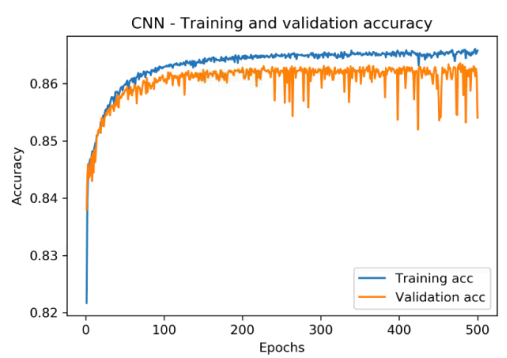

Figure 12: Validation accuracy against epochs for CNN

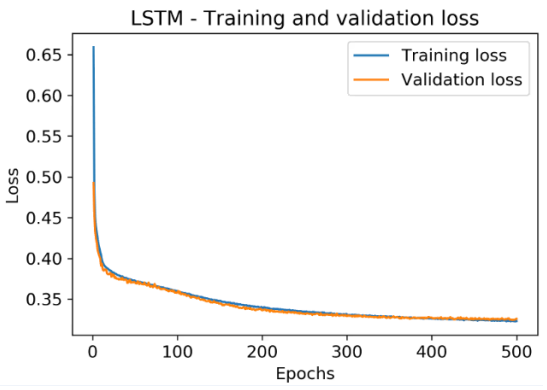

Figure 13: Validation loss against epochs for LSTM

a time-series dataset, compare and report the performance of this model on these two domains.

Since our implemented approach showed a significant attacks detection capability and demonstrated that performance of attack 


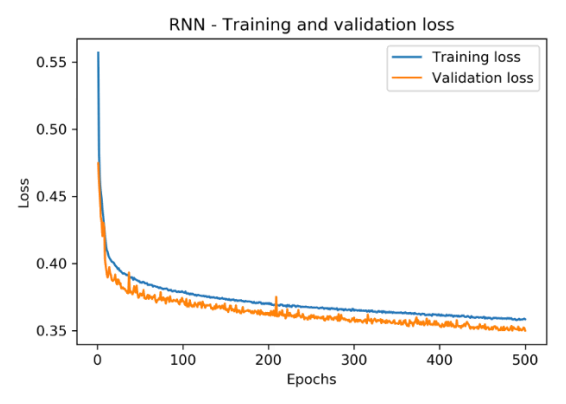

Figure 14: Validation loss against epochs for RNN

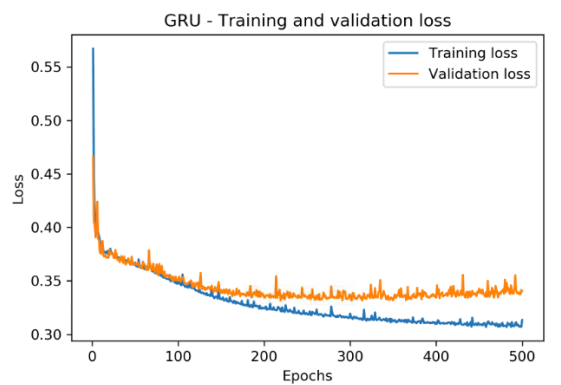

Figure 15: Validation loss against epochs for GRU



Figure 16: Validation loss against epochs for CNN

detection techniques applied can be influences by the nature of network transactions with respect to the domain of application. As suggested that the robustness and resilience of operational CPS state and performance are influenced by the safety and security measures in place which is specific to the CPS device in question. Hence the need for further investigation in diverse domain in other to ascertain this claim. The authors are currently engaged in work in this domain.

\section{REFERENCES}

[1] Abdelrahman Ayad, Mohsen Khalaf, and Ehab El-Saadany. 2018. Detection of false data injection attacks in automatic generation control systems considering system nonlinearities. In 2018 IEEE Electrical Power and Energy Conference (EPEC). IEEE, 1-6.

[2] Lorena Cazorla, Cristina Alcaraz, and Javier Lopez. 2016. Cyber stealth attacks in critical information infrastructures. IEEE Systems fournal 12, 2 (2016), 1778-1792.

[3] Thomas Chen and Saeed Abu-Nimeh. 2011. Lessons from stuxnet. Computer 44 4 (2011), 91-93.
[4] Global Energy Cyberattacks. 2011. Night dragon. McAfee Foundstone Professional Services and McAfee Labs (2011).

[5] Hope Eke, Andrei Petrovski, and Hatem Ahriz. 2020. Handling minority class problem in threats detection based on heterogeneous ensemble learning approach. International journal of systems and software security and protection 11, 2 (2020).

[6] Tom Fawcett. 2006. An introduction to ROC analysis. Pattern recognition letters 27, 8 (2006), 861-874.

[7] Martin T Hagan, Orlando De Jesús, Roger Schultz, L Medsker, and LC Jain. 1999. Training recurrent networks for filtering and control. In Recurrent neural networks: Design and applications. CRC press, 311-340.

[8] Guo Haixiang, Li Yijing, Jennifer Shang, Gu Mingyun, Huang Yuanyue, and Gong Bing. 2017. Learning from class-imbalanced data: Review of methods and applications. Expert Systems with Applications 73 (2017), 220-239.

[9] Brendon Harris and Ray Hunt. 1999. TCP/IP security threats and attack methods. Computer communications 22, 10 (1999), 885-897.

[10] Kevin E Hemsley, E Fisher, et al. 2018. History of industrial control system cyber incidents. Technical Report. Idaho National Lab.(INL), Idaho Falls, ID (United States).

[11] Abdulmalik Humayed, Jingqiang Lin, Fengjun Li, and Bo Luo. 2017. Cyberphysical systems security-A survey. IEEE Internet of Things fournal 4, 6 (2017), $1802-1831$.

[12] USDHS ICS-CERT. 2014. ICS-monitor incident response activity; Information Sharing. National Cybersecurity and Communications Integration Center (2014).

[13] Hyung Seok Kim, Jae Min Lee, Taerim Park, and Wook Hyun Kwon. 2000. Design of networks for distributed digital control systems in nuclear power plants. In Intl. Topical Meeting on Nuclear Plant Instrumentation, Controls, and Human-Machine Interface Technologies (NPIC\&HMIT 2000). Citeseer.

[14] Stefan Kombrink, Tomáš Mikolov, Martin Karafiát, and Lukáš Burget. 2011. Recurrent neural network based language modeling in meeting recognition. In Twelfth annual conference of the international speech communication association.

[15] Robert M Lee, MJ Assante, and T Conway. 2017. CRASHOVERRIDE: Analysis of the threat to electric grid operations. Dragos Inc., March (2017).

[16] Ondrej Linda, Todd Vollmer, and Milos Manic. 2009. Neural network based intrusion detection system for critical infrastructures. In 2009 international joint conference on neural networks. IEEE, 1827-1834.

[17] Victoria Marquis, Rebecca Ho, William Rainey, Matthew Kimpel, Joseph Ghiorzi, William Cricchi, and Nicola Bezzo. 2018. Toward attack-resilient state estimation and control of autonomous cyber-physical systems. In 2018 Systems and Information Engineering Design Symposium (SIEDS). IEEE, 70-75.

[18] Thomas H Morris, Zach Thornton, and Ian Turnipseed. 2015. Industrial control system simulation and data logging for intrusion detection system research. 7 th annual southeastern cyber security summit (2015), 3-4.

[19] Artem A Nazarenko and Ghazanfar Ali Safdar. 2019. Survey on security and privacy issues in cyber physical systems. (2019).

[20] NJCCIC. 2017. CRASHOVERRIDE NJCCIC Threat Profile, official site of the state of new jersey Original Release Date: 2017-08-10 and accessed on 16/07/20. NFCCIC (2017). https://www.cyber.nj.gov/threat-center/threat-profiles/ics-malwarevariants/crashoverride

[21] A Odewale. 2018. Implementing secure architecture for industrial control systems. Proceedings of the 27th COREN Engineering Assembly, Abuja, Nigera (2018), 6-8.

[22] Joseph Slowik. 2019. Evolution of ICS Attacks and the Prospects for Future Disruptive Events. Threat Intelligence Centre Dragos Inc (2019).

[23] Yanmin Sun, Andrew KC Wong, and Mohamed S Kamel. 2009. Classification of imbalanced data: A review. International journal of pattern recognition and artificial intelligence 23, 04 (2009), 687-719. 